\title{
Reviving Healthcare Delivery: Context-Based Issues for Revamping Primary Healthcare in Nigeria
}

\author{
Owumi, B.E. Taiwo, P.A. Kolo, V.I. \\ Department of Sociology, University of Ibadan
}

\begin{abstract}
The relationship between health and sustainable development have long been established. Hence the need to rigorously pursue strategies to improve the health status of a nation's citizenry through functionally effective and efficient delivery systems. However, health and healthcare delivery is a major problem confronting developing nations like Nigeria. In August 1987, Primary healthcare (PHC) was adopted as the cornerstone of health care and health policy in Nigeria. About three decades after, studies have shown that health and healthcare in Nigeria have not significantly improved; and the delivery of health services have become a perennial problem, defying the various solutions that had been advanced to solving it over time. In the face of dwindling economic reality in Nigeria, reviving primary healthcare becomes a viable alternative that will not only produce healthy and productive citizens, thereby generating national wealth; but will also reduce the cost of health care delivery to the average Nigerian. This paper presents a theoretical diagnosis of the state of affairs of healthcare delivery in Nigeria focusing on PHC as the hub of health policy. It examines the problems of PHC, identifying gaps within it that militates against successful implementation and outcomes. The paper concluded that certain deficits in the scope and policy process of PHC in Nigeria accounted for the programme's incapacitation. It however recommended a thorough situation analysis as a prelude to a bottom-up policy approach, inclusion of the informal health sub-sector especially the traditional health care practitioners as a viable as well as cost effective strategy for the revamping of primary health care in Nigeria.
\end{abstract}

Keywords: Health, primary, context-based healthcare, policy, revamping

DOI: $10.7176 / \mathrm{JHMN} / 58-10$

\section{Introduction}

The phenomenon of health and illness in human history dates back to time immemorial. They constitute some of the realities that man has had to face in his experience of life. While health on the one hand describes a state of normalcy in which the entire human person including his body and mind as one system, are able to function optimally due to balances between man's biological composition as well as other and external psycho-social factors that affect his life; illness is a deviation from, or an interruption to such normal order. It refers to any condition that alters the state of man's health by disrupting the hitherto existing balance, through various causes including diseases. Hence, man from prehistoric times had also devised means of maintaining health and restoring the body to normalcy at whenever he falls sick. This culminated in the emergence of traditional medicine as can be found among different peoples and cultures.

However, due to complexities in social life particularly in the $17^{\text {th }}-18^{\text {th }}$ centuries, modernization and the consequent formalization of social organization, traditional medicine which used to be the major source of healthcare delivery seem to have been swept aside by western medicine, due to the emphasis on 'germ theory' of disease and driven by modern medical science and technology.Increasingly, healthcare began to appropriate towards centralization, as national governments started to play greater roles in the provision of health and associated welfare services to citizens. There was therefore the need to develop means vis-à-vis policies, strategies and funding, through which healthcare can be made available to the people. This gave birth to the notion of healthcare delivery systems - an arrangement consisting of "all organizations, people and actions whose primary intent is to promote, restore or maintain health (WHO, 2007). In most developing countries, modern medicine was introduced by the colonizers, such as Britain in the case of Nigeria. Due to different sociopolitical and economic climes that obtain in different countries of the world, healthcare delivery also has various character which varies across countries. Like other sectors in developing countries, health is also characterized by various forms of inadequacies posed by political economy among other factors. Nigeria has struggled over the years, to develop and sustain different policies and programmes for the delivery of care to its vast citizenry. However, all of these attempts have suffered the same fate as developmental efforts in other spheres of national life. The aim of this paper is to provide critical insights and raise major issues for the revamping of primary health care - the hub of the country's health policy, for the overall revival of health care delivery in Nigeria.

\section{Healthcare Delivery In Nigeria: Situational Analysis}

In every country of the world, the health sector is very key to the functional operation of the nation as a complex body of system. This is due to the important roles that the sector plays in promoting and maintaining the healthcare need of the people who ultimately constitute productive agents whose activities culminate in national 
development. Although Nigeria's is not an exception to this principle, the government has not accorded health and healthcare delivery the much required attention in the area of prioritization, funding and various other input commitment. This has resulted in the unfavourable state of affairs that characterize the delivery of healthcare in the country. Unlike many other countries, Nigeria is highly heterogeneous, polarized along various socio-cultural divides. Each of the peoples and cultures making up Nigeria had developed various indigenous methods of meeting their healthcare needs from time immemorial. However, the advent of colonialism led to the 'importation' of western medicine, initially to meet the need of the colonial officials. This culminated in the establishment of the first hospital (Sacred Heart hospital, Lantoro) in Abeokuta, present day Ogun state in the year 1895 . With this, alien western medicine gradually began to exist side-by-side the indigenous medical systems in meeting the healthcare needs of Nigerians. The situation has changed with countless numbers of various western facilities which has spread throughout the country, providing various health services to the people. By the year 2005, the Federal Ministry of Health $(\mathrm{FMoH})$ estimated a total of 23,640 health facilities in Nigeria, $85 \%$ of which are primary health care facilities, $14 \%$ secondary and $0.2 \%$ tertiary (Oyibocha, Irinoye, Sagua, Ogungude-Essien, Edeki and Okome (2014). Apart from urban-bias in the siting of the facilities (Jegede, 2002), the personnel, organizational and material equipment of the facilities have reduced them to the status of mere consulting rooms (Jegede, 2002).

Although western medicine is the officially recognized system of health care in Nigeria, traditional medicine has also continued to thrive and compete favourably with its western counterpart, gaining popularity among more than 70\% of Nigerians (Adefolaju, 2010).Nigeria's health sector is domiciled in, regulated and controlled by the federal ministry of health which shares responsibility with ministries of health at the state and local government levels, since health is a concurrent item in the Nigerian constitution (Erinosho, 1998). Healthcare delivery which is organized along the three major levels of care: primary, secondary and tertiary, is however ridden by a myriad of problems which incapacitated the system to a state of virtual collapse (Eme, Uche and Uche, 2014). Rather than preventive medicine which forms the thrust of Primary health care - the country's health policy, the health sector and healthcare delivery in Nigeria has derailed towards curative medicine which is morecostly and unsustainable, given the fragile economic base on which Nigeria rests. The major problems of healthcare delivery in Nigeria include inadequate supply of physicians, poor distribution of health personnel and health facilities, high cost of healthcare service, shortage of drugs, poor remuneration of health workers, attitude of health works, infrastructural deficit, and low community involvement in, and awareness of health programmes (Jegede, 2002; Abdulraheem, Olapipo and Amodu, 2012). These have not only translated to low quality of care delivery, but has further enshrined inequality in access to health services among Nigerians. Hence, access to healthcare in Nigeria is largely determined by the ability to pay, a situation that has resulted in medical tourism among the bourgeois class (Audu, Ojua, Ishor and Abari, 2013). In the face of economic crisis and pervasive poverty which affects more than half of Nigeria's population especially people in rural areas which constitute more than $70 \%$ of the country's population, the implication is that majority of Nigerians lack the wherewithal to afford healthcare and its associated costs (United Nations Development Program [UNDP], 2007). Little wonder, the country's health indicators (life expectancy at birth, maternal mortality rate, infant and neo-natal mortality rates and deaths from notifiable diseases have gone from bad to worse, particularly in the last two decades (Oyibocha, Irinoye, Sagua, Ogungide - Essien, Edeki and

Okome, O.L., 2014). This development is largely due to lack of prioritization as well as negligent attention that is accorded primary health care, which is the hub of health care delivery in Nigeria (Abiodun, 2010).

\section{Concept and relevance of primary healthcare}

In climes such as obtain in developing countries of which Nigeria is one, there is need to develop systems that can meet the healthcare need of the large and vulnerable populations. It was in the bid to achieve this, particularly in the face of communicable diseases that ravaged the country at that point in time and their associated outcomes, that primary healthcare was introduced. Basically, primary healthcare hinges on the idea of preventive rather than curative medicine. It aims at providing preventive, promotive, curative and rehabilitative services through proximate access to health care for majority of a country's population using a grass root approach (Olise, 2007). PHC serves as the first contact of patients with the health system and therefore uses a 'nip in the bud approach' to address health problems. Primary healthcare is aimed at preventing the occurrence of health problems in the first place, and where such problems occur, provide immediate care where and when problems arise. This explains why primary healthcare is largely domiciled at the local government level, despite the fact that health in Nigeria is a concurrent item, constitutionally. It is to bring healthcare closer to the people where they live and work, on individual, household or community basis. From conception, this aimed at producing a comprehensive health care system, based on promotion, protection, prevention, restoration and rehabilitation of the health of citizens within the available resources. In a nutshell a primary health care center or facility is a small unit which provides a family with the health services other than those which can only be provided in a hospital. 
Officially introduced by the WHO 1978 Alma Ata declaration, primary healthcare became the overall National Health Policy of Nigeria from August 1987 when it was introduced. According to WHO (1978), Primary health care is essential health care based on practical, scientifically sound and socially acceptable methods and technology made universally accessible to individuals and families in the community through their full participation and at a cost that the community and country can afford to maintain at every stage of their development in the spirit of self-reliance and self-determination. From this definition, it can be inferred that primary healthcare was intended, not just to focus on priority areas of health needs, but also to be culturally sensitive, community participatory, sustainable and self reliant.

Intended to affect the entire national population, its (PHC's) main stated objectives included accelerated health care personnel development; improved collection and monitoring of health data; ensured availability of essential drugs in all areas of the country; implementation of an Expanded Programme on Immunization (EPI); improved nutrition throughout the country; promotion of health awareness; development of a national family health program; and widespread promotion of oral rehydration therapy for treatment of diarrheal disease in infants an)d children. (Nigeria primary healthcare Policies. (library of congress country studies; CIA world fact book))

Realizing this therefore required PHC to be inter-sectoral, while appropriating inputs from education, agriculture, livestock, finance, information. Thus, a combination of workforce from different disciplinary background, is needed if the programme must operate efficiently and effectively, in both preventive and curative aspects of healthcare delivery at the community level.

The goals of PHC as captured above was borne out of the recognition of health as a fundamental right which must be provided if people must function to optimum capacityand be considered productive. However, due to unfavourable social structure (social inequality) health care was unaccessible to many people, especially those from developing countries. In Nigeria for instance where more than $70 \%$ of people are located in rural areas with predominantly low income population (Abdulraheem, Olapipo and Amodu, 2011), the case is not different in this regard. As such, primary healthcare can be said to be well suited for a country like Nigeria where the population is large and poverty level is very high since the gap between the rich and the poor is wide. If adequately designed and properly implemented, the role and relevance of primary healthcare in achieving universal access to healthcare will be well felt by the citizens. It is the inadequacy in design, scope and implementation of the programme as well as its consequent unsustainability that has led to its incapacitation.

\section{Primary Healthcare: Challenges And Prospects In Nigeria}

Before Nigeria's independence in 1960, a 10-year developmental plan (1946-1956) was introduced to enhance health care delivery. This led to the establishment of various health schools and institutions, several clinics and health centers, all of which consolidated by the mid 1980s produced tremendous developments in health care progress with the creation of over 10, 000 facilities including general hospitals and several other health centers (Awosika, 2005). Another programme, the basic health services scheme (BHSS) was developed and operated between 1975 and 1980. Although the BHSS eventually failed due to various reasons, including poor commitment on the part of the federal ministry of health, poor funding and refusal of health workers to work in the rural areas, the scheme provided viable foundations on which PHC came to be operate when it was officially launched in 1988 vis-à-vis the National health policy of Nigeria (Alenoghena, Aigbiremolen, Abejegah and Eboreime, 2014; Lambo, 2015). The full implementation of PHC in Nigeria commenced in 1992, using the LGAs as fulcrum of operation, marking the beginning of decentralization of the health system (Cueto, 2005), particularly along the three tiers of government (federal, state and local government). In the same year, the national primary healthcare development agency was created as the coordinating structure charged with mobilizing support, both local and international, for the operation and sustainability of PHC

Alenoghena, Aigbiremolen, Abejegah and Eboreime (2014) identified five key strategies by which primary healthcare operated in Nigeria:

4.1. Community mobilization: this involved sensitizing the people on PHC and the opportunities it presents in terms of healthcare services vis-a-vis the need to utilize such opportunities. In addition to resource mobilization, cost minimization and appropriate utilization of health services, Magawa (2012) noted that community mobilization engenders community participation and community ownership, and ultimately guarantees sustainability of health programmes

4.2. Service integration: this involves the incorporation of different services together under a single platform, for the purpose of maximizing health resources while improving efficiency and effectiveness. An example is the integration of child health with maternal health programmes/services for wider impact and coverage.

4.3. Health research: this is a strategy that enables the identification of health problems and their epidemiology in any given population. With proper research, resources can be appropriately deployed 
and newer methods also can be developed for solving health problems so identified. As a strategy of PHC, health research produces an evidence-based approach to programme implementation.

4.4. Capacity building: the importance of manpower in any programme cannot be overemphasized. It is on this realization that PHC adopted capacity building as a strategy for equipping workers and managers with requisite skills, using a continuous training and re-training approach. This is done through workshops and seminars that are organized for health workers on regular basis.

4.5. Non-governmental and international collaborations: the responsibility of designing and implementing a large programme as PHC cannot be borne by government alone, given the huge burden of the venture. As a strategy, PHC therefore accommodates inputs from other sectors outside of the government. These include both local and international development partners and NGOs such as UNICEF, USAID, DFID Save the Children, Doctors Without Borders, Pathfinders International, Faithbased organizations, and so forth. These collaborations have impacted tremendously on PHC by strengthening the existing resource base of government and thereby the scope of the programme. In specific terms, they have donated funds and materials, provided personnel and organized training in support of PHC in Nigeria.

These strategies initially contributed to the implementation of PHC in no little ways. Tremendous progress was made based on the devolution of healthcare to better involve the LGAs. Infrastructure was massively developed and community health workers' training institutions were established, which began to churn out large numbers of health personnel. The programme also recorded $80 \%$ immunization coverage for children under 5years as well as improvement in measles coverage from 10\% in 1984 to 85\% in 1990 (Lambo, 2015). However, PHC's success story was short-lived with the eventual incapacitation of the programme due to certain shortcomings in the localization of PHC to the Nigerian context, challenges in the design and implementation of the policy as well as inadequate measures for sustaining the programme. Apart from the programmes lack of legal backing, which has implications for the force with which the programme can operate, the withdrawal of support (non-governmental and international collaborations) by donor organizations largely due to the unpopular regime of Gen. Sanni Abacha (Lambo, 2015) further undermined the already attained feat of PHC in Nigeria.

\section{Factors Responsible For The Incapacitation Of Phc In Nigeria}

5.1. Lack of political will: Successive Nigerian governments have not shown adequate commitment to the pursuing the vision of PHC. Approval and release of funds for routine activities of the programme has continued to be politicized which greatly affects the realization of the goals of PHC. As observed by Alenoghena et al (2014), there has been greater emphasis on the construction of gigantic physical structures compared to the provision of good health services. The situation is worsened by political instability occasioned by frequent change in government which also affects the sustainability of the programmes, since successive governments in Nigeria have a traditional penchant for novel achievements regardless of what neglect of existing programmes might portend for the nation. Most successes recorded in the implementation of PHC such as the eradication of poliomyelitis are attributable to the intervention of development partners such as Rotary international not the government as would have been expected.

5.2. Inadequate Financing: given the centrality of adequate funding to the success of any project, it will be apt to conclude that inadequate financing spells doom for PHC. Whereas WHO recommends that at least $5 \%$ of gross national product should be committed to the healthcare, Nigeria and most developing countries still fail to meet with the standard even when developed countries commit as high as $10 \%$ of GNP to healthcare (Olise, 2007). The implication of this is that PHC is starved of funds requisite for its proper functioning, since the LGAs themselves are struggling to survive on internally generated revenue. This also implicates unavailability of materials and facilities as well as poor condition of service for health workers which according to Lambo (2015) is another factor for the failure of PHC, particularly in the rural areas where the bulk of the problems reside.

5.3. Poor synergy between the collaborating sectors: PHC was intended to be operated on a multidisciplinary basis, vis-à-vis collaboration between different sectors: government and non-governmental as well as health sector and other relevant sectors of national life such as education, agriculture, ecology, water, industry and housing (Obionu, 2007). Poor synergy between these sectors have resulted in improper coordination as well as waste of resources due to duplication of efforts. There were instances where two different agencies with varied mission refused to share information even when the need arises (Alenoghena et al, 2014).

5.4. Poor community participation: from its conception in the Alma Ata declaration (1978) of WHO, PHC was intended to be community-oriented, such that the people themselves are active partners in the provision of their own healthcare. The document described community participation as the process by which individuals and families assume responsibility for their own health and welfare and for those of 
the community, and develop the capacity to contribute to their community development. Communities participate by assisting in problem identification and other aspects of health research, as well as other methods of support for PHC. One important potential of community participation lies in its ability to engender sustainability and better utilization of the PHC services. The breakdown of mutual support between the government and the community also contributed to the problems of primary health care.

5.5. Deficit in personnel: personnel or health workers are the pillar of PHC. They extent of personnel required for effective implementation of PHC depends largely on the scope and coverage the programme, including the national population size which changes over time. Hence, there is need to match PHC with commensurate workforce suitable for the scope of healthcare need; and also ensure equitable distribution of health workers based on need. This has not been the case. Rather, PHC in Nigeria has suffered from problems ranging from inadequacy of personnel, unequitable distribution of available personnel, inter-cadre conflicts, poor job satisfaction (Alenoghena et al, 2014).

\section{Revamping Primary Healthcare In Nigeria: Context-Based Issues}

The above factors largely account for the defeat of PHC's aims and objectives in Nigeria. However, there is great need to revamp the programme, given its centrality to the to healthcare delivery and its associated impacts on national development. Scholars have identified various strategies for improving PHC and healthcare delivery generally. The following approaches are recommended as strategies that will better address the problem of PHC, going by the peculiarities of the Nigerian context and its challenges:

6.1. Strong commitment to public health education and health research: A major problem of healthcare delivery and utilization in Nigeria is the lack of commitment to or prioritization of education and research, particularly in the area of health and health care. Proper importance should be accorded not only the formal education, but also the informal education of the general Nigerian, using the community as pivot. Such education should include awareness creation about common health problems, their potential implications/burden and how to prevent their incidence. This will instill in people a realization of the need to promptly seek help as and when they have health problems. Ultimately, massive education such as is being advanced will ill-perception about PHC services and facilities by bridging the gap between indigenous culture and 'alien' primary healthcare, thereby promoting care utilization. Similarly, health research must be intensified first to provide deeper understanding of health problems, while identifying their root causes. Promoting health research especially those that are epidemiologyoriented will help generate data without which policy and planning must not be made. The research process should also aim at identifying stakeholders such as community heads, renown traditional medical practitioners and religious leaders, whose domain are though outside of the formal health sector, but can influence the peoples' perception and utilization and ofcourse, the sustainability of PHC. Indeed, intensive research vis-à-vis situational analysis is indispensable to the proper revamping of sustainable PHC in Nigeria.

6.2. Focus on preventive rather than curative care: preventive care refers to measures that are taken to keep diseases and other health problems at bay, in order to guide against their occurrence in the first place. Curative care on the other hand involves the use of therapeutics, including diagnostic and treatment approaches in the management of health problems. As the saying goes, 'prevention is better than cure'. Focus on preventive rather than curative care will reduce the burden on the already fragile health infrastructure, minimize costs and ultimately improve the quality of life of the people, with relatively less resources. Improvement in nutrition, sanitation, water and other public health strategies are indispensable in this regard (Jegede, 2002). Individuals, households and communities should be empowered through public health education, to 'produce' their own health so that they also play vital roles as active partners in the overall PHC, which also has implications for the sustainability of the programme.

6.3. Development of traditional medicine vis-à-vis medical pluralism: the very concept of PHC provides a place of importance to the indigenous medical system of communities. However, the programme in Nigeria has not accorded traditional medicine and its associated science, the relevance that it deserves, particularly in a clime where western medicine is welcome with much distrust and suspicion, and therefore low level of utilization especially in the rural areas (Olise, 2007). There is need to recognize the role and strength of traditional medical system which itself has functioned as a viable PHC for centuries (Subedi, 1992). Due to weak resource base, modern medicine should only be used as a supplement for traditional medicine under an arrangement of medical pluralism, guided by cooperation rather than competition between practitioners of the two systems. For instance, the skills of traditional medical personnel such as birth attendants, bone setters and psychiatrist can go a long way in addressing the personnel problems especially in the rural areas where the acceptance and utilization of modern services is a major issue itself. However, care must be taken to localize or contextualize the 
operation of traditional medicine as part of the PHC programme due to variation of its practice across peoples and culture (Subedi, 1992). Again, proper research would be required in this case, especially from the dimension of the social sciences.

6.4. Monitoring and evaluation: the collapse of PHC did not happen suddenly or overnight. It gradually unfolded in a cumulative, instalmental process incapacitation which was not observed until the virtual wreck of the programme. The routine and periodic check, retreat and stock-taking which monitoring and evaluation (M\&E) offers was either absent or too minimal to impact the programme which partly accounts for the undesirable state of PHC affairs that now dawns on the country. The national primary healthcare development agency (NPHCDA) in conjunction with external M\&E experts should be engaged in the monitoring and evaluation of the programme, apart from the managers at various sections of the programme. Care must be taken to ensure multi-disciplinarity and multi-sectoriality in the M\&E process. This will help ascertain effectiveness and efficiency while improving strategy at every stage deemed necessary, and at the same time assuring of quality of service through the checks of the M\&E process.

6.5. Improved financing, efficient and effective utilization of funds: starvation resulting from inadequate financial commitment and/or proper utilization of funds has been a major cause of programme failure in Nigeria. Proper funding is very key to the successful implementation of PHC. Funds are needed to acquire materials (drugs, vaccines, vehicles), staff manpower and drive the programme. Special attention must be paid to making the remuneration of health workers attractive, in order to motivate them, particularly those in the rural areas. Efforts must also be made to make the programme as costfree as possible; given the generally high level of poverty and its implication for healthcare utilization. Conscious efforts must however be made and checks put in place, to ensure that the funds are not embezzled as is typical of most projects in Nigeria.

6.6. Training and inclusion of Patent medicine sellers into the programme: Patent medicine sellers or vendors who operate outside the formal health sector constitute a good but neglected bulk of workforce with huge potentials for the revamping of PHC in Nigeria, especially in the area of coverage. This category of healthcare providers is easily available and accessible to provide care for people which is relatively affordable and flexible, sometimes characterized by home service (Amzat and Razum, 2014). Since they are business men and women, many of them operate as itinerant vendors and enjoy high level of confidence and popularity in their community of operation. Although their operation is sometimes characterized by malpractices, they are strategically posited to contribute immensely to the revamping of PHC in Nigeria, if only they can be trained, legally incorporated and regulated as part of the scheme.

\section{Summary And Conclusion}

The discussion thus far presents an overview of Nigerian health policy: PHC and the problems militating against the attainment of its goals. Although PHC possess strategies that could have changed the face of healthcare and healthcare delivery, the problem lied in its localization to the Nigerian context on the one hand, and the way and manner it was implemented, on the other hand. Specific challenges and shortcomings of the PHC programme in Nigeria were identified, and recommendations were advanced on how the issues can be best addressed, taking into account the peculiarities of the Nigerian socio-cultural scene. Institutional and legal recognition was advocated for traditional medicine and patent medicine sellers who have been unrecognized stakeholders whose role in the delivery of healthcare to the Nigerian peoples cannot be overemphasized. The paper therefore argued that the duo be incorporated into the formal PHC system, while consolidating on research, training, improved funding and proper supervision vis-à-vis proper M\&E. In conclusion, PHC remains a viable tool for effective and efficient healthcare delivery in Nigeria. However, there is great need to re-visit the programme with a view to revamping it, as a launching pad to overhauling the entire health sector. Ultimately, the development and institutionalization of medical pluralism is a highly recommended for improvement in the quality and utilization of healthcare in Nigeria.

\section{REFERENCES}

Abdulraheem I. S., Olapipo A. R. and Amodu M. O. 2012.Primary health care services in Nigeria: Critical issues and strategies for enhancing the use by the rural communities

Abiodun AJ (2010). Patients ${ }^{\text {ee }}$ Satisfaction with Quality Attributes of Primary Health Care Services in Nigeria. J. Health. Manag., 12(1): 39-54.

Alenoghena, I., Aigbiremolen, A.O., abejegah, C. and Eboreime, E. 2014. Primary health care in nigeria: strategies and constraints in implemntation.International Journal of Community Research http://www.arpjournals.com

Amzat, J. and Razum, O. 2014. Medical Sociology in Africa. London: Springer 
Audu, D.T., Ojua, T.A., Ishor, D.G., and Abari, C.A., 2013. Inequality and Class Difference in Access to Healthcare in Nigeria. Research on Humanities and Social Sciences vol.3, No.16, 45

Awosika L. Health insurance and managed care in Nigeria. Ann Ibadan Postgrad Med. 2005;3:40-6

Eme, O.I., Uche, O.A., and Uche, I.B. 2014. Building a Solid Health Care System in Nigeria: Challenges and Prospects. Academic Journal of Interdisciplinary Studies Vol 3 (6)

Erinosho, O.A. 1998. Health sociology for universities, colleges and health-related institutions. Ijebu-Ode: Bulwark Press

Jegede, A.S. 2002. Problems and prospects of healthcare delivery in Nigeria: issues in political economy and social inequality. In Isiugho-Abanihe, U.C., Isamah, A.N. and Adesina, J.O. (eds) Currents and perspectives in sociology. Lagos: Malthouse press Limited

Lambo, E. 2015. Primary health care: realities, challenges and the way Forward. A paper delivered at the 1st annual primary health care Lecture of the National Primary Health Care Development Agency (NPHCDA) on tuesday, 8th December, 2015 at the Shehu Musa Yar'Adua Centre, Wuse Zone 4, Abuja

Nigeria Primary Health Care Policies. (The Library of Congress Country Studies; CIA World Factbook)Retrieved from http://www.photius.com/countries/nigeria/society/nigeria society primary health care $\sim 10006 . h t m l$ on $18^{\text {th }}$ July, 2016 at $11: 16$ am

Obioha, E.E. and Molale, M.G. 2011. Functioning and Challenges of Primary Health Care (PHC) Program in Roma Valley, Lesotho. Ethno Med, 5(2): 73-88 (2011)

Oyibocha, E.O., Irinoye, O., Sagua, E.O., Ogungide - Essien, O.T., Edeki, J.E. \& Okome, O.L. 2014. Sustainable Healthcare System in Nigeria: Vision, Strategies and Challenges.IOSR Journal of Economics and Finance (IOSR-JEF) Volume 5 (2) Sep.-Oct. 2014, PP 28-39

Stephen Gillam: Is the Declaration of Alma Ata still relevant to primary health care? BMJ, 8 March 2008, Volume 336 pp536-538

Subedi, J. 1992. Primary healthcare and medical pluralism exemplified in Nepal: a proposal for maximizing healthcare benefits. Journal of sociological focus. Vol 25 (4) special issue: health and healthcare in developing countries

WHO 1978. Primary health care. Geneva, World Health Organization, 1978. 